\title{
Actuator management for ECRH at ASDEX Upgrade
}

\author{
Christopher J. Rapson ${ }^{\mathrm{a}}$, Matthias Reich ${ }^{\mathrm{a}}$, Joerg Stober ${ }^{\mathrm{a}}$, Wolfgang Treutterer ${ }^{\mathrm{a}}$, and the ASDEX Upgrade team \\ ${ }^{a}$ Max Planck Institute for Plasma Physics, Boltzmannstrasse 2, 85748 Garching, Germany
}

\begin{abstract}
Automated actuator management will be necessary on long pulse fusion experiments to adjust to unforeseen plasma events and unpredictable actuator availability. However, as a control problem, actuator management is underdeveloped in the fusion community. This contribution proposes an algorithm based on a control hierarchy and a cost function to optimally allocate scarce actuator resources to various objectives in real-time. Details are given on the development and offline testing which have been completed ready for deployment at ASDEX Upgrade. Electron Cyclotron Resonance Heating (ECRH) is particularly relevant for actuator management due to its localised deposition which can flexibly target specific regions of the plasma for different effects such as non-inductive current drive, impurity regulation, control of MHD modes and of course heating. A further motivation is that automated actuator management will simplify the setup of ECRH, in keeping with the long term goal of integrating MHD control as a routine part of ASDEX Upgrade experiments.
\end{abstract}

Keywords: control system, real time, actuator management, automation, ECRH, DCS, ASDEX Upgrade

\section{Introduction}

At fusion experiments today, physicists typically program a pulse schedule to a high level of detail, specifying the timing of individual actuators to achieve their physics goal. However, there is a growing realisation that this approach is not well suited to long pulse experiments since actuator availability and plasma conditions can not be predicted before the pulse. Even at ASDEX Upgrade (AUG), with pulse lengths $<10 \mathrm{~s}$, unexpected actuator failures and plasma events lead to a significant fraction of pulses not reaching their physics goals.

On long pulse experiments and fusion power plants, it will be preferable to prescribe high level parameters [1] and allowable deviations [2]. An abstraction layer would automatically convert these specifications to detailed actuator commands in a way that is optimal given the plant and plasma conditions at the time. These concepts are very much in line with those of segmented pulse schedules and exception handling [3, 4].

One important component of the abstraction layer is actuator management. Electron Cyclotron Resonance Heating (ECRH) is a particularly good candidate for actuator management due to its flexibility. Power deposition is localised and can be steered in real-time (RT)

Email address: chris.rapson@ipp.mpg.de (Christopher J. Rapson)

Preprint submitted to Fusion Engineering and Design from near the plasma centre to near the edge, with current drive in either direction. This allows ECRH to be used for heating the plasma, driving non-inductive current, expelling high-Z impurities from the core and controlling MHD modes such as sawteeth, Neoclassical Tearing Modes (NTMs) of various mode numbers and Edge Localised Modes (ELMs) [e.g 5, 6, 7, 8, 9]. ECRH actuator management is complex, since it can not be assumed that one gyrotron is replaceable by another arbitrary gyrotron. For example, one launcher could be aimed at the centre and another at the edge of the plasma. In addition, the resources are limited.

As a control problem, actuator management for fusion is at an early stage of development, despite its perceived importance for ITER [10]. Automated actuator management is used extensively in fields such as aerospace [e.g. 11, 12] and automotive [e.g. 13, 14] control, and several concepts have been proposed by the fusion community $[15,16]$. The work presented here uses one of these concepts known as a control hierarchy, with high level controllers delegating suitable objectives to low level controllers. Hierarchies can extend over multiple levels. The concept is similar to cascaded control, but should not be confused with supervisory control [4]. Control hierarchies have the advantage of being modular and reusing existing simple controllers. To our knowledge, this will be the first implementation of a control hierarchy in a fusion experiment.

September 26, 2014 
Table 1: There are $\left(n_{\text {gyr }}\right)^{n_{\text {target }}}$ ways to allocate $n_{\text {gyr }}$ gyrotrons to $n_{\text {target }}$ targets. This can be visualised by indexing the list in base $n_{\text {target }}$. Here an example for 2 gyrotrons and 3 targets.

\begin{tabular}{ccc}
\hline \hline combination index & target for gyr0 & target for gyr1 \\
\hline $0_{10}=00_{3}$ & 0 & 0 \\
$1_{10}=01_{3}$ & 0 & 1 \\
$2_{10}=02_{3}$ & 0 & 2 \\
$3_{10}=10_{3}$ & 1 & 0 \\
$4_{10}=11_{3}$ & 1 & 1 \\
$5_{10}=12_{3}$ & 1 & 2 \\
$6_{10}=20_{3}$ & 2 & 0 \\
$7_{10}=21_{3}$ & 2 & 1 \\
$8_{10}=22_{3}$ & 2 & 2 \\
\hline \hline
\end{tabular}
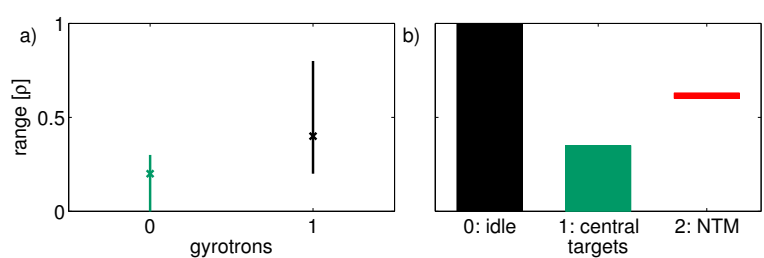

Figure 1: a) Deposition location and range of movement for gyrotrons. b) Extent of targets. In this paper, $\rho$ refers to $\rho_{\text {poloidal }}$, the normalised radial magnetic flux co-ordinate.

An additional motivation is related to the long term goal for NTM control to become a routine part of the experimental program at AUG [17]. NTM control is now reaching a level of maturity where it is being trialled on other scenarios. However, this requires a specialist to select ECRH settings for RT control, and hence is used sparingly. The algorithm presented here takes many of the complex characteristics of ECRH into account and should allow NTM control to be integrated in any scenario without consulting a specialist.

This paper presents a description of a novel algorithm in section 2 with details on RT deployment and results from offline testing in section 3.

\section{Description of Algorithm}

An Actuator Management (AM) module is added to the control chain to decide which gyrotron is best suited to which task, and potentially which tasks will not be addressed. Commands are passed down the hierarchy to pairs of low-level controllers for gyrotron power and launcher steering. Existing low-level controllers developed for NTM control [18] can be re-used.

To illustrate the decision logic of the AM, consider a simplified example with 2 gyrotrons and 2 targets central heating and NTM control. The targets will be augmented by a third option, which is for the gyrotron

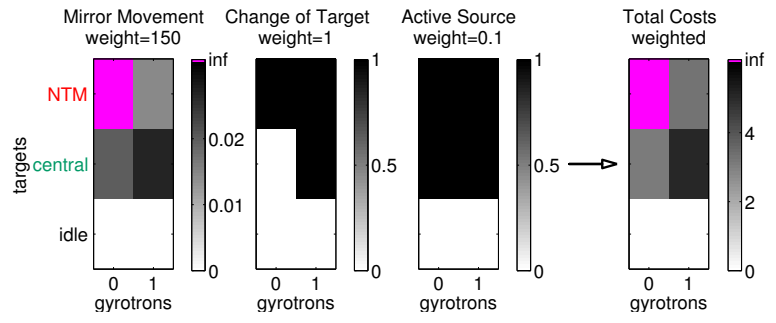

Figure 2: Costs for each gyrotron when allocated to each target.

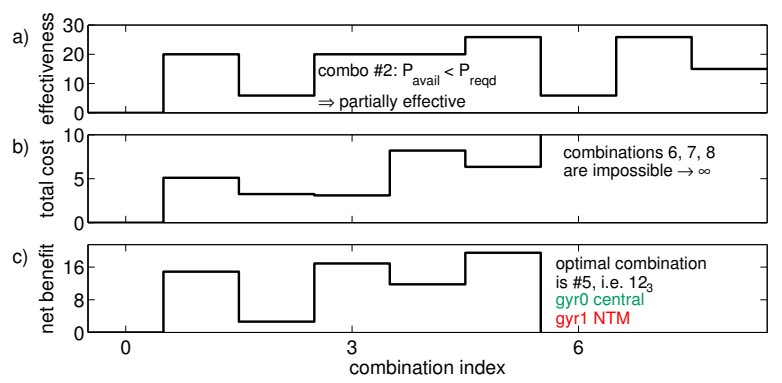

Figure 3: Effectiveness, total costs and net benefit for all possible ways to allocate 2 gyrotrons to 3 targets.

to be idle. Table 1 shows all possible combinations that gyrotrons can be assigned to targets. The example will start from the initial conditions shown in fig. 1, where gyr0 is assigned to central heating and gyr1 is idle.

An analogy can be made to the "Weapons Target Assignment" problem [19]. The goal is to identify an optimal assignment of gyrotrons (weapons) to regions of plasma (targets) to maximise the net benefit (expected damage). As for all assignment problems, it scales badly with the number of inputs. For a small number of gyrotrons and targets, it is feasible to calculate the net benefit for all possible combinations by brute force.

The net benefit is formulated as a cost function, comparing the effectiveness of ECRH to the total of 3 costs:

- movement cost: actuator effort to steer the launcher

- switching cost: a form of hysteresis to discourage switching near decision boundaries

- active cost: a penalty for each non-idle gyrotron, to minimise resource use

Each of these costs has a weighting which allows them to be tuned relative to each other. They are then added together to give a total cost, as shown in fig. 2. Infinite movement cost indicates that this target is out of range for this particular gyrotron. Idle gyrotrons have no cost. The total cost matrix can then be converted into a vector of combinations, shown as axes (b) of fig. 3 .

Costs can be regarded as independent and added lin- 
early, but the effectiveness of ECRH at a particular target is nonlinear. Each target requires a certain amount of power, but applying more than required adds no further benefit. Applying less than the required power is generally much less effective and should therefore be penalised. The final parameter in the calculation is the target importance, allowing some targets to be prioritised over others. The pseudocode to calculate the effectiveness $e$ of a given combination is

$$
\begin{aligned}
& e=0 \\
& \text { for } j=0 \text { to }\left(n_{\text {targets }}-1\right) \\
& P_{\text {normalised }, j}=\frac{\sum_{k=0}^{n_{g y r}-1} P_{\text {available }, k} *\left(\text { target }_{k}==j\right)}{P_{\text {required }, j}} \\
& \text { if } P_{\text {normalised, } j} \geq 1 \\
& e+=\text { importance }_{j} \\
& \text { else } \\
& e+=\text { importance }_{j} * P_{\text {normalised }, j}^{\text {penalty_exp }} *\left(1-\text { penalty_factor }^{\text {poration }}\right.
\end{aligned}
$$

Finally, the cost is subtracted from the effectiveness and the combination with the highest net benefit is selected, as shown in fig. 3. In this case, gyr0 would remain on central heating and gyr1 would be switched to NTM control, even though it is expected to be only partially effective. Note that applying both gyrotons to central heating does not improve effectiveness, since 1 is usually enough to expel high-Z impurities at AUG [5].

There are a few unusual circumstances which are worth noting. If a gyrotron is active, but not under the jurisdiction of the AM, its power may contribute to one of the targets. Changing its target is of course not allowed. Conversely, if a gyrotron is not available it will have 0 effectiveness but finite costs, therefore the optimal combination will include this gyrotron being idle.

\section{Deployment of RT Algorithm}

After initial development and testing in Matlab ${ }^{\circledR}$, including closing the loop with a model of the launcher dynamics (not shown), the algorithm has been deployed in the DCS framework [20]. As such, it can take advantage of the quality tags which are delivered with each input signal and extensive configuration capabilities. The AM can be configured to control any number of gyrotrons and targets, with settings for cost weights, penalty_exponent, penalty_factor, required power per target and nominal power per gyrotron. Target importances are provided as preset combinations:

i central heating only
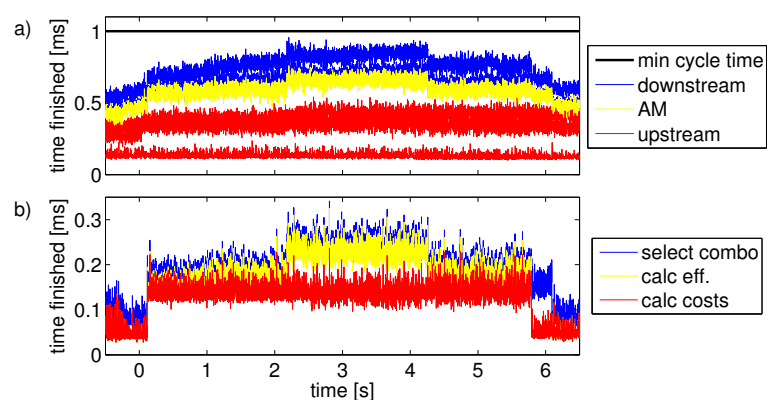

Figure 4: RT performance using archived data from shot \#30569 with 4 gyrotrons and 6 targets. a) Time of completion for modules in the DCS execution chain compared to the minimum cycle time as a deadline. b) Breakdown of the AM execution time by function.

ii central heating and tracking rational q-surfaces to pre-empt NTMs

iii central heating and reacting when NTM amplitude exceeds a threshold

iv combination of $i i$ and $i i i$

The configuration for AUG uses 4 gyrotrons and 6 targets giving 1296 combinations. The computer has a lot to do, but fig. 4a) shows that in tests using real archived data, the RT requirements were met. The AM is data driven, i.e. it begins computation as soon as all signals from upstream threads are available. Once it publishes its outputs there are two further downstream threads which must be finished before the end of the DCS cycle. The chain of threads is finished in $<0.95 \mathrm{~ms}$ and hence with a buffer to DCS' shortest cycle time of $1 \mathrm{~ms}$.

Within the AM, profiling shown in fig. 4b) indicates that calculating costs is the most intensive part of the algorithm. Other functions save time since infeasible combinations are eliminated early - if they have infinite costs then there is no point in calculating effectiveness.

The output of the AM is shown in fig. 5 for 2 different shots, and 2 different modes for each shot. Note that these are not closed loop simulations, and hence the algorithm has no influence over the movement of the launchers. At each time step, the AM indicates to which target the gyrotrons would have been allocated.

In \#30645, gyr1 was under repair and hence unavailable for the whole shot while a trip of gyr3 was detected at $t=2.5 \mathrm{~s}$. The RT equilibrium is invalid until $t=0.5 \mathrm{~s}$, so all target locations and deposition locations are also invalid and the low level controllers' modes are set to idle. From $t=0.5-0.7 \mathrm{~s}$ there is no $q=1.5$ surface in the plasma, so both gyr2 and gyr3 are allocated to the $q=2.0$ surface. Gyr0 is set to central heating and stays there for most of the shot. Once the $q=1.5$ surface appears, 1 gyrotron is allocated to each of the $q=1.5$ and $q=2.0$ surfaces, de- 

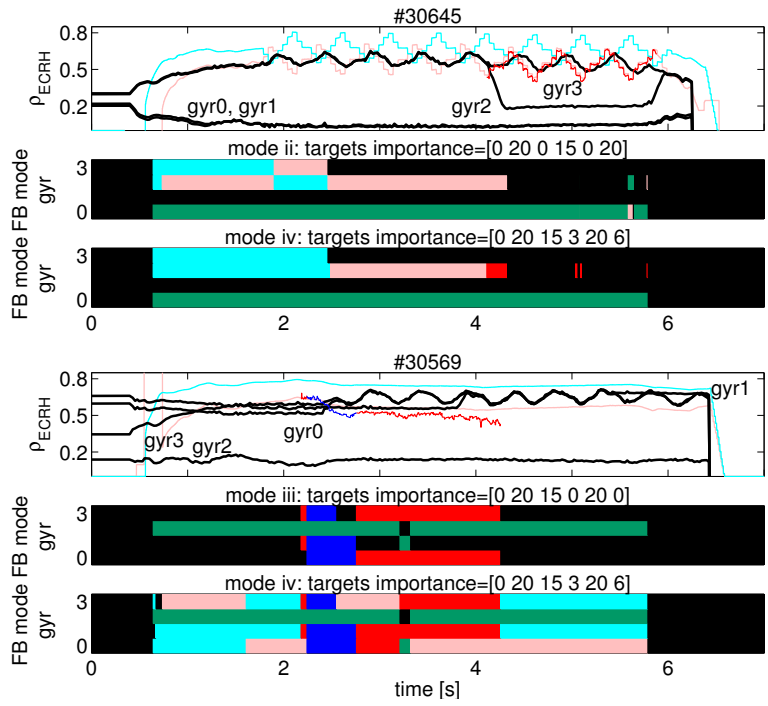

Figure 5: Results from replaying archived data with shot number and AM mode given in the axes titles. Black lines show the deposition location of gyrotrons from RT ray tracing. Coloured lines show the location of targets. 'Central' is at $\rho=0$. The NTM location is only shown when an NTM is detected in RT. Coloured blocks show which target a gyrotron was allocated to at which time. The colour code indicates the relevant target: black is idle, green is central, red is a $3 / 2$ NTM, light red is the $q=1.5$ surface, blue is a $2 / 1$ NTM and light blue is the $q=2.0$ surface. Importances are given in the same order: [idle, central, $3 / 2, q=1.5,2 / 1, q=2.0]$.

pending on which is nearer. Oscillations in the q-surface positions were added artificially in many shots during this campaign for physics reasons, unrelated to actuator management. Since the NTM target has 0 importance, the appearance of an NTM from $t=4.1-5.9 \mathrm{~s}$ had no effect. Gyr 2 continued tracking the $q=1.5$ surface until the launcher is moved far away. At $t=5.6 \mathrm{~s}$, the $q=1.5$ surface is estimated to be in range of gyr0 (but not gyr2) for a short time. Estimating the range is difficult because it is a nonlinear function of the range of the linear actuator, which is limited for safety reasons. In addition, the estimate is affected by noisy measurements, as seen here since the range estimate is only briefly beyond the $q=1.5$ surface. In closed loop operation, blips such as these could lead to gyrotrons being steered towards and trapped in non-optimal configurations, so this behaviour will have to be monitored when the AM is deployed. Increasing the weight of the switching cost is one option to prevent such occurrences.

In comparison, when the target importances are modified (one axes lower in fig. 5), the relative importance of preventing the 2/1 NTM increases, and 2 gyrotrons are allocated to follow the $q=2.0$ surface, until one of these gyrotrons trips. Since the settings for required power to prevent 2/1 NTMs are well above the available power, the effectiveness penalty is large and it is more optimal to allocate the gyrotron to the $q=1.5$ surface. When the NTM is detected, this gyrotron is switched to attempt to stabilise it, as long as the NTM is within range.

In \#30569, all 4 gyrotrons were available, with only gyr2 experiencing a short trip at $t=3.2 \mathrm{~s}$. When the AM is in mode $i i$, the gyrotrons are only activated when an NTM is detected. A $3 / 2 \mathrm{NTM}$ is detected at $t=2.2 \mathrm{~s}$ and a $2 / 1$ NTM at $t=2.3 \mathrm{~s}$. 2 gyrotrons are deemed sufficient for the $3 / 2$ NTM, but 3 are required for the $2 / 1$ NTM, as long as all 3 are in range. During the short trip of gyr2, its role is taken over by the idle gyr1.

If the AM is set to mode $i v, 2$ gyrotrons are allocated to pre-empt the $2 / 1 \mathrm{NTM}$ and 1 gyrotron is allocated to pre-empt the $3 / 2$ NTM in the periods where no NTM is detected. During the period where an NTM is detected, the allocation is similar to the example with mode iii. In mode iii, gyr1 replaces gyr2 during its short trip which incurs only 1 switch cost. In mode $i v$, either option requires 2 gyrotrons to be switched and since gyr0 is marginally closer, the optimal combination is different.

Since the AM algorithm has passed all offline tests, it is now ready for testing on an AUG plasma experiment.

\section{Conclusions \& Future Work}

A novel algorithm has been developed using a cost function to determine the optimal allocation of gyrotrons to targets. The allocation is designed as a controller hierarchy by selecting controller modes for pairs of controllers for gyrotron power and launcher mirrors. The algorithm is implemented as part of the DCS framework and is hence highly configurable and robust to degraded inputs. Offline tests have been completed on archived data and the algorithm is ready for online testing in AUG's autumn 2014 campaign.

In the future, a $q=1$ target will be added for sawtooth control, current drive should be taken into account and the algorithm could potentially be parallelised. Online results will be monitored to tune settings if necessary. If it can be shown that settings are universally valid, then this routine will simplify the setup of ECRH for physicists and help to make MHD control a more routine part of AUG operation.

The algorithm presented is just one example of automated actuator management, which so far has not received much attention in the fusion control community. Significant advances and experimental proof will be required in the coming years to optimally exploit longpulse devices. 


\section{Acknowledgements}

This work has been carried out within the framework of the EUROfusion Consortium and has received funding from the European Union's Horizon 2020 research and innovation programme under grant agreement number 633053. The views and opinions expressed herein do not necessarily reflect those of the European Commission.

\section{References}

[1] H. Riemann, et al., Experiment planning using the high level parameter concept, Fusion Eng. Des. 85 (2010) 478 - 481. Proceedings of the 7th IAEA Technical Meeting on Control, Data Acquisition, and Remote Participation for Fusion Research.

[2] R. Nouailletas, et al., Plasma discharge management for longpulse tokamak operation, Fusion Sci. Technol. 64 (2013) 13-28.

[3] A. Winter, et al., Towards the conceptual design of the ITER real-time plasma control system, Fusion Eng. Des. 89 (2014) $267-272$. Design and implementation of real-time systems for magnetic confined fusion devices.

[4] G. Raupp, et al., Real-time exception handling - use cases and response requirements, Fusion Eng. Des. 87 (2012) 1891 1894. Proceedings of the 8th IAEA Technical Meeting on Control, Data Acquisition, and Remote Participation for Fusion Research.

[5] D. Wagner, et al., Progress and first results with the new multifrequency ECRH system for ASDEX Upgrade, Plasma Science, IEEE Transactions on 37 (2009) 395-402.

[6] T. P. Goodman, et al., Real-time multi-EC-actuator MHD control on TCV, AIP Conference Proceedings 1580 (2014) 161166.

[7] F. Felici, et al., Integrated real-time control of MHD instabilities using multi-beam ECRH/ECCD systems on TCV, Nucl. Fusion 52 (2012) 074001.

[8] D. Moreau, et al., Integrated magnetic and kinetic control of advanced tokamak plasmas on DIII-D based on data-driven models, Nucl. Fusion 53 (2013) 063020.

[9] M. Reich, et al., NTM stabilization experiments at ASDEX Upgrade, in: Europhysics Conference Abstracts, Proceedings of the 40th EPS Plasma Physics Conference, Helsinki (2013) Paper P2.151, 2013.

[10] A. Winter, et al., Present Status of the ITER Real-time Plasma Control System Development, in: 2012 18TH IEEE-NPSS REAL TIME CONFERENCE (RT), IEEE; Nucl and Plasma Sci Soc (NPSS); Univ Calif, IEEE, 345 E 47TH ST, NEW YORK, NY 10017 USA, 2012. 18th IEEE-NPSS Real Time Conference (RT), Univ Calif, U S Dept Energy, Lawrence Berkeley Natl Lab (Berkelly Lab), Berkeley, CA, JUN 09-15, 2012.

[11] J. Paradiso, Adaptable method of managing jets and aerosurfaces for aerospace vehicle control, Journal of Guidance Control and Dynamics 14 (1991) 44-50. CONF ON GUIDANCE, NAVIGATION, AND CONTROL, BOSTON, MA, AUG 14-16, 1989.

[12] P. Tsiotras, H. Shen, C. Hall, Satellite attitude control and power tracking with energy/momentum wheels, Journal of Guidance Control and Dynamics 24 (2001) 23-34.

[13] D. Bianchi, A. Borri, B. Castillo-Toledo, M. Di Benedetto, S. Di Gennaro, Smart management of actuator saturation in integrated vehicle control, in: Decision and Control and European Control Conference (CDC-ECC), 2011 50th IEEE Conference on, 2011, pp. 2529-2534. doi:10.1109/CDC. 2011.6161087.
[14] A. Sciarretta, L. Guzzella, Control of hybrid electric vehicles, Control Systems, IEEE 27 (2007) 60-70.

[15] D. Humphreys, et al., Novel aspects of plasma control in ITER, Phys. Plasmas submitted for publication in Aug. 2014 (2014).

[16] W. Treutterer, et al., Architectural concept for the ITER plasma control system, Fusion Eng. Des. (2014) -.

[17] M. Reich, et al., Real-time control of NTMs using ECCD at ASDEX Upgrade, in: Submitted to Proceedings of the 25th IAEA Fusion Energy Conference, St. Petersburg, Russia, 2014.

[18] C. Rapson, et al., Simulation of feedback control system for NTM stabilisation in ASDEX Upgrade, Fusion Eng. Des. 88 (2013) 1137 - 1140. Proceedings of the 27th Symposium On Fusion Technology (SOFT-27); Liége, Belgium, September 2428, 2012.

[19] Wikipedia, Weapon target assignment problem wikipedia, the free encyclopedia, 2014. URL: http: //en.wikipedia.org/w/index.php?title=Weapon target_assignment_problem\&oldid=618386081, [Online; accessed 8-September-2014].

[20] W. Treutterer, et al., ASDEX Upgrade Discharge Control System - a real-time plasma control framework, Fusion Eng. Des. 89 (2014) 146 - 154. Design and implementation of real-time systems for magnetic confined fusion devices. 